\title{
The Research of Structural Damage Identification via Wavelet-Genetic Simulated Annealing Algorithm
}

\author{
Deqing Guan ${ }^{1 a}$, Qinchang Wang ${ }^{2 b}$,Yungang Liu ${ }^{3 c}$ \\ ${ }^{1,2,3}$ College of Civil Engineering, Changsha University of Science \& Technology, Changsha, China \\ a491596452@qq.com, b976371682@qq.com, c1601376939@qq.com
}

\begin{abstract}
Keywords:damage identification;wavelet analysis; simulated annealing algorithm; modal parameter Abstract.Due to disadvantages of premature convergence and poor local optimization, a structural damage identification based on wavelet-genetic stimulated annealing algorithm(abbreviated to SAGA) was proposed.Firstly, wavelet analysis is applied to identify the structural damage location and the number of damaged unit.Then, the objective function is established by using the natural frequencies and modal shapes of the structure, the damage degree of the structure is taken as the design variable. The quantification of the structural damage were performed with genetic-stimulated annealing algorithm. The combination of wavelet analysis and genetic simulated annealing algorithm not only makes up for the deficiency of the two methods, but also reduces the unknown quantity after the wavelet analysis determines the damage location.Finally, a numerical simulation of a continuous beam model was given. The result showed that the damage location and the degree of the structure can be identified well.The effectiveness of the proposed method was verified.
\end{abstract}

\section{Introduction}

In the process of long-term, due to environmental erosion, material aging, loads ,misuse and so on, the structure will inevitably appear different degree of damage.If any damage is accumulated without repairing it, it will eventually lead to destruction of the whole structure or loss of function.Therefore, it is of great theoretical and practical significance to find the damage of structure and to accurately identify the damage location and degree before the damage has been expanded.

Wavelet analysis is a milestone in the development of Fourier transform. The concept of wavelet analysis was first proposed by the French scholar J.Morlet in the early 1980s. Wavelet analysis is more widely used and is a better signal analysis method.For nonstationary signal analysis, wavelet analysis is of great advantage.In time domain and frequency domain, wavelet analysis can show the local characteristics of the signal well.Raghavendrachar used wavelet transform of the curvature mode to identify damaged concrete continuous beam model, it is proved that the wavelet transform is reliable to local damage ${ }^{[1]}$.Deqing Guan adopted the continuous steel box beam as the numerical simulation object $^{[2]}$. The strain mode parameters of the damaged beam are obtained by modal analysis. The wavelet analysis is used to carry out the continuous wavelet transform of the strain mode parameters. The wavelet coefficient shows the maximum modulus at the crack, and the damaged location can be detected by the maximum modulus. This article proves the validity of the method.Koh identified the structural parameter by the local search function of the genetic algorithm ${ }^{[3]}$. When Metropolis studied the two-dimensional phase transition in 1953, the idea of simulated annealing algorithm was first proposed.In view of this situation, the combination of genetic and annealing algorithm can obtain improved genetic simulated annealing algorithm.Genetic algorithm are used to generate the initial population and continue genetic manipulation to find the optimal solution ${ }^{[4]}$.Combined with the strong local optimization of annealing algorithm and the ability to get rid of local traps, accepted the elimination of the inferior solution of genetic algorithm by a certain probability ${ }^{[5]}$, and used the cooling process to control the operation of the whole algorithm, SAGA can quickly find the optimal solution in the global range ${ }^{[6]}$.

\section{The Principle of Wavelet-Genetic Simulated Annealing Algorithm}

Wavelet Transform. Wavelet transform is a convolution of a signal with an analysis window (mother 
wavelet) shifted in time and dilated by a scale parameter. If a $\psi(t) \in L^{1}(R) \cap L^{2}(R)$ and $\psi(0)=0$, the function $\psi_{a, b}(t)$ is defined as Eq. (1).

$$
\psi_{a, b}(t)=\frac{1}{\sqrt{|a|}} \psi\left(\frac{t-b}{a}\right) \cdot
$$

where $\psi(t)$ is called basic wavelet , $\mathrm{t}$ is the variable in real number field $\mathrm{R}$, In solving the problems, the continuous wavelet transform must be invertible.So the convolution equation of continuous wavelet transform is defined as Eq. (2).

$$
\mathrm{WT}_{f}(a, b)=\left\langle f(t), \psi_{a, b}(t)\right\rangle=\frac{1}{\sqrt{a}} \int_{-\infty}^{+\infty} f(t) \psi *\left(\frac{t-b}{a}\right) d t .
$$

where $\mathrm{a}$ is the scale dilation parameter that determines the width of the wavelet, $\mathrm{b}$ is the translation parameter corresponding to the position of the wavelet, and $\psi^{*}(t)$ is the complex conjugate function of $\psi(t)$.All the singular points on the signal function are recognized by the modulus maxima of the wavelet transform.According to theory of mechanics, $\frac{d^{2} w\left(v^{+}\right)}{d x^{2}} \neq \frac{d^{2} w\left(v^{-}\right)}{d x^{2}}$ is derived from $E\left(v^{+}\right) \neq E\left(v^{-}\right)$. It indicates that the second derivative of the basic vibration mode of damage cross section is discontinuous.In the field of structural damage,the singular point of the signal is the same as structural damage location.

Damage Degree Identification Based on SAGA.[1]The source of simulated annealing algorithm is the similarity between complex combinatorial optimization problem and solid annealing process.During the heating process, the temperature of the solid slowly rises, then the temperature drops a little bit.In the process of rise, the ions in the solid are disordered and the internal energy increases.As the temperature drops, the ions slowly tend to an orderly state and reach a state of equilibrium at every moment.Finally, the ground state is reached at normal temperature and the internal energy is reduced to a minimum.Different initial temperature have different total number of iteration and final solution ${ }^{[7]}$.Determination of initial temperature should be optimized by considering other parameters, synthetically. The value must be able to ensure that the algorithm can get out of the local trap and make the problem as accessible as possible to all possible solutions.In the identification of the degree,this paper selects the exponential cooling method according to the nature and characteristics of the objective function. The change of temperature is related to parameters $a$, and values of parameter directly determine the cooling process, $a \in[0.5,0.99]$.In the article, $a$ can be taken as 0.95.[2]Crossover and Variation operation.Crossover is the operation of forming two new individuals by exchanging two pairs of paternal chromosomes with each other in a certain way and crossover probability.The recommended range is 0.6 1.0.The variantion is to replace some of the genes in the chromosome coding sequence with other alleles in the gene locus, resulting in a new individual.The average value is between 0.005 and 0.1.[3]Objective function. When the structure is damaged, it's regarded as decrease of stiffness, while the quality hardly changes.Therefore, it's not necessary to consider the change of quality. The damaged degree of the unit is simulated only by the reduction of stiffness. The modulus of stiffness of the unit is expressed as $\beta$. The stiffness of the destroyed unit is $\beta E I$, and $\beta \in[0,1] ; \alpha$ is the degree of damaged unit, $\alpha=1-\beta$.In actual measurement, there is a large error in the identified result due to the inaccuracy of the vibration data.Based on the frequency and modal shape,the objective function is established by taking the weighted sum of the frequency error function and modal shape error function as the objective, as following Eq. (3).

$$
J=F_{\omega} \sum_{i=1}^{m}\left(\frac{f_{i}^{\text {test }}-f_{i}{ }^{c a l}(\alpha)}{f_{i}^{\text {test }}}\right)^{2}+F_{\phi} \sum_{i=1}^{n} \sum_{j=1}^{k}\left(\phi_{i j}^{\text {test }}-\phi_{i j}^{c a l}(\alpha)\right)^{2} .
$$

In the equation, $J$ is the objective function; $\alpha$ is the design variable, the degree of damage to the structure; $F_{w}, F_{\phi}$ is a weighted factor of frequency and vibration type,In paper, $F_{w}, F_{\phi}$ set to $1 ; f_{i}^{\text {test }}, f_{i}^{\text {cal }}$ is 
the structure of the measurement and calculation of the $\mathrm{i}$ order of modal frequency; $\varphi_{i j}^{\text {test }}, \varphi_{i j}^{\text {cal }}$ is the structure of the measurement and calculation of the $\mathrm{i}$ order of modal vibration mode; $\mathrm{k}$ is the number of nodal displacement;m means the order of frequency;n indicates the order of vibration mode.[4]The formation and acceptance mechanism of the new solution. The simulated annealing algorithm adopts the Metropolis criterion to accept the new solution. In addition to the optimized solution, the method can accept the bad solutions based on probability.The algorithm only accept less bad solutions, so that it jump out from local optimal solution. The expression of probability is as shown in Eq. (4).

$$
p=\exp \left(\frac{-\left[J\left(\alpha_{j}\right)-J\left(\alpha_{i}\right)\right]}{0.5 T}\right) \cdot
$$

Where $\alpha_{j}, \alpha_{j}$ are the current solution and new solution of algorithm; $J\left(\alpha_{i}\right), J\left(\alpha_{j}\right)$ are the objective function values corresponding to the current solution and the new solution;the constant $\mathrm{k}$ is 0.5 .If $J\left(\alpha_{j}\right)-J\left(\alpha_{i}\right)$ is less than or equal to 0 , the new solution $\alpha_{j}$ is accepted;If the value is greater than 0 , then the new solution is determined by probability; The computer randomly produces a number, and when its value is less than or equal to probability, the new solution is used as current solution and vice versa.[5]The Algorithm Termination Criteria.The final value is usually determined by the termination criteria.The commonly used criteria are the termination criteria proposed by Kirkpatrick.If the resulting solution does not change, the algorithm is terminated.The final temperature set to 20 .When the temperature drops to $20^{\circ} \mathrm{C}$, the algorithm stops running and outputs the final solution.

The basic flow chart of genetic annealing algorithm is shown in Fig. 1.

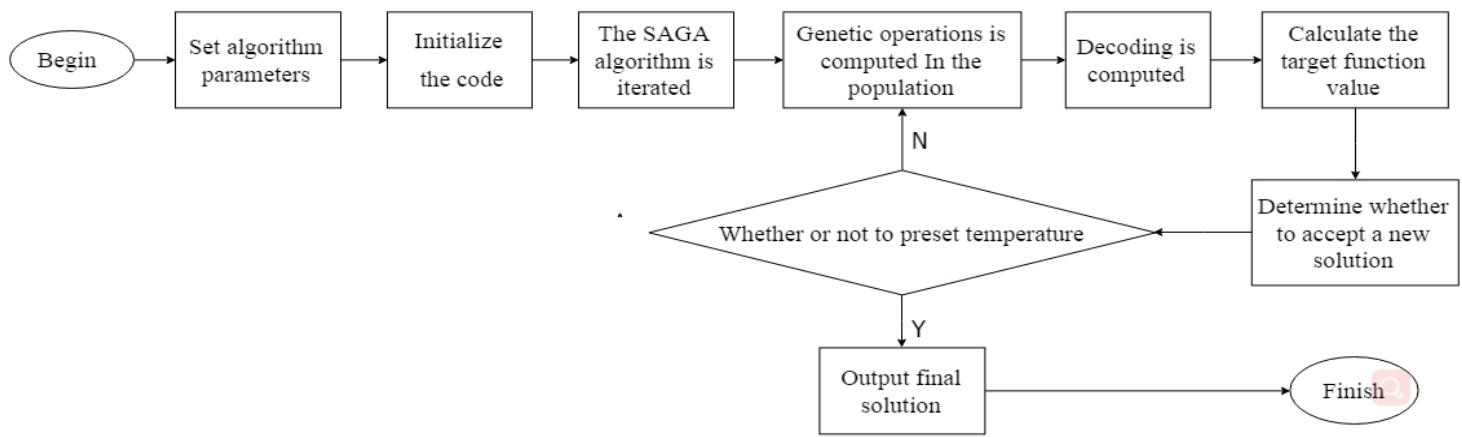

Fig. 1. The flow chart of genetic simulated annealing algorithm

\section{Numerical Simulation Analysis}

Damaged Condition.A two-span continuous beam structure finite element model which contains damage shown in Fig. 2. Sectional dimension: $b \times h=250 \mathrm{~mm} \times 300 \mathrm{~mm}$ Modulus: $E=207 \times 10^{11} \mathrm{~N} / \mathrm{m}^{2}$;Poisson's ratio: ${ }^{\mu}=0.3$;Density: $\rho=7800 \mathrm{~kg} / \mathrm{m}^{3}$; Span:6000mm. The whole structure is equally divided into 60 units.Structural damage was simulated by reducing element stiffness. Damaged units were assumed in article, as shown in Table 1.

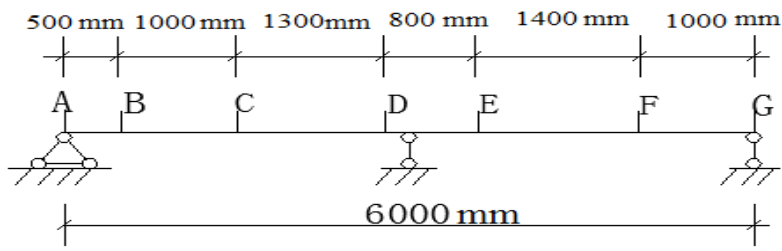

Fig. 2. Continuous beam finite element model

\begin{tabular}{|ccc|}
\hline Cases & Location & Extent \\
\hline & [Unit 5] & $6 \%$ \\
Condition 1 & [Unit 15] & $2 \%$ \\
& [Unit 28] & $4 \%$ \\
& [Unit 50] & $8 \%$ \\
\hline
\end{tabular}

Table 1. Continuous beam damage cases

Damaged Location Identification.The finite element model of the structure was established, and the damage is supposed in unit 5 (B), unit 15 (C), unit 28 (D) and unit 50 (F). The parameter of the rotation modal are obtained by modal analysis, and the Gaussian wavelet is selected as the basic wavelet.The rotation modal is taken as an object to carry out the continuous wavelet transform.Then wavelet coefficients of the condition 1 are calculated,as shown in Fig.3. It's observed that the location 
of the singular point in the figure under condition 1 corresponds to the location of the damaged unit in the continuous beam.Four damage that indicate a consistent hypothesis.

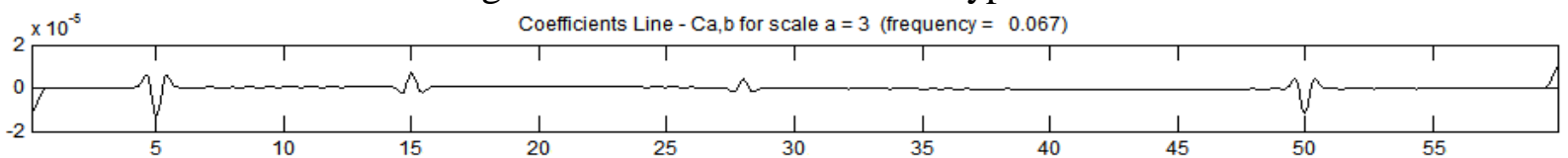

Fig. 3. Wavelet coefficient of continuous beam with four damage

Identification of the Degree of Structural Damage by SAGA.Using wavelet analysis, the number of damaged unit only is four in unit5, unit15, unit28 and unit 50.After repeated calculation and analysis, the relevant parameters are set.The population size:50;Crossover probability:0.8; Variation probability:0.1; Initial temperature value:60; Cooling coefficient:0.95. The optimal change of population is shown in Fig. 4.Final damage identification results are shown in Fig. 5.

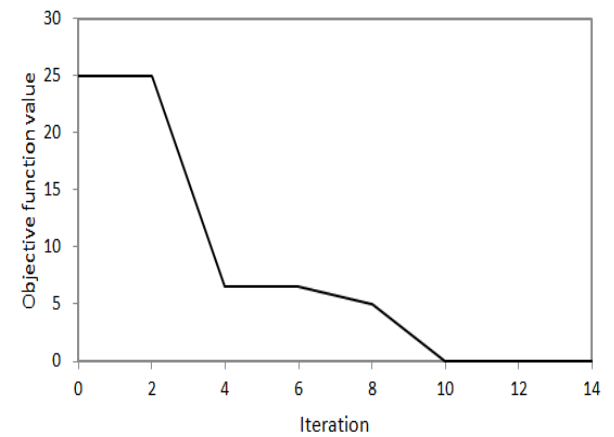

Fig. 4. Iterative optimization curve

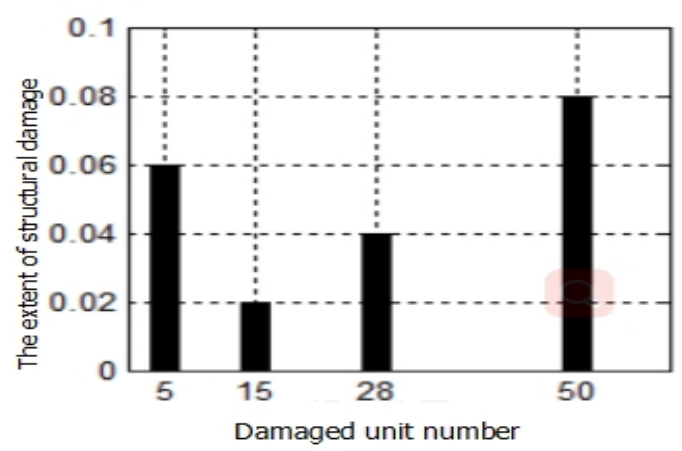

Fig. 5. Damage identification results

The number of average iteration is 10.4 times, and the running time is 275 seconds. The algorithm accurately identifies unit5, unit 15,unit28, unit50, and the damage degree respectively is $6 \%, 2 \%, 4 \%$, $8 \%$.The results are the same as assumed damage of continuous beam structure.

\section{Conclusions}

1.The concept of wavelet-genetic simulated annealing algorithm has been proposed and applied successfully in the field of structural damage identification. This method not only can accurately determine the location of structural damage, but also can effectively quantify the degree of damage. 2.The genetic simulated annealing algorithm has excellent robustness and operability. It can identify the damage degree of the structure accurately, and its convergence speed is faster. The SAGA is a more reliable and effective intelligent algorithm, which has the ability of stronger global and local search.

3.The numerical simulation results indicate the effectiveness of wavelet-genetic annealing algorithm in identifying structural damage. This method has good applicability,tremendous reference value for damage identification and small damage of complex structures in civil engineering.

\section{Acknowledgements}

This work was financially supported by the National Natural Science Foundation of China (51378079).

\section{References}

[1] Raghavendrachar M, Akan A E:Journal of Structural Engineering,American Society of Civil Engineering Vol. 118 (1992),p. 2186

[2] Deqing Guan :Journal of Architecture and Civil Engineering Vol. 27 (2010), p. 21(In Chinese)

[3] Koh C G,Zhao S L, Liaw C Y: NED for Health Monitoring and Diagnostics ,San Diego(2002)

[4] Jenkins W M: Journal of Structural Engineering Vol. 118(1992), p. 3103 
[5] Shao P F: Computer Engineering \& Applications Vol. 43 (2007), p. 62

[6] Botello S, Marroquin J L, Oñate E, et al:International Journal for Numerical Methods in Engineering Vol. 45 (2015), p. 1069

[7] Jeong I K, Lee J J:Engineering Application of Artificial Intelligence Vol. 9(1996), p. 523 